\title{
CrystEngComm
}

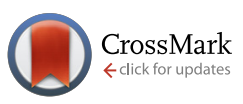

Cite this: CrystEngComm, 2016, 18 5869

Received 11th March 2016,

Accepted 17th June 2016

DOI: $10.1039 / c 6 c e 00561 f$

www.rsc.org/crystengcomm

\section{Polymorphic transformations in glycine co- crystals at low temperature and high pressure: two new examples as a follow-up to a glycine- glutaric acid study $\dagger$}

\author{
E. A. Losev, ${ }^{\star a b}$ B. A. Zakharov ${ }^{a b}$ and E. V. Boldyreva ${ }^{a}$
}

\begin{abstract}
The effects of temperature and pressure on the co-crystals of glycine with DL-tartaric and phthalic acids (GTa and GPh, respectively) have been studied by X-ray diffraction and Raman spectroscopy in comparison with those on glycine-glutaric acid (GGa). On cooling, no phase transitions were observed in GTa or GPh, in contrast to the situation with GGa. On hydrostatic compression, both GTa and GPh underwent reversible phase transformations, accompanied by fracture. In the high-pressure phases, the main structural framework was preserved, and the number of crystallographically independent molecules in the unit cell increased. In GTa, dimers are squeezed together so that some hydrogen bonds get a three-centered character, and the interactions of one of the two glycine molecules change dramatically.
\end{abstract}

\section{Introduction}

Interest in studying and understanding the effects of temperature and pressure on multicomponent molecular crystals has grown considerably in recent years. ${ }^{1}$ Their properties differ from those of the corresponding single-component crystals and often cannot be predicted due to unique lattice structures. This has rendered the prediction of the properties of multi-component materials very challenging. Of particular note are the difficulties in predicting structural strain anisotropy, the possibility of structural phase transitions, ${ }^{2-6}$ or the potential for co-crystals to form salts via proton transfer. ${ }^{7-12}$

Analysis of the Cambridge Structural Database (version 5.36 from November 2015$)^{13}$ reveals ten two-component crystals of glycine with carboxylic acids (excluding hydrates and solvates), namely, three salts with oxalic acid, ${ }^{14-16}$ salts with malonic, $^{17}$ maleic, ${ }^{18}$ 3-nitrophthalic ${ }^{19}$ and 2-hydroxy-5sulfobenzoic $^{20}$ acids, as well as co-crystals with glutaric, ${ }^{21,22}$ phthalic $^{23}$ and DL-tartaric ${ }^{24}$ acids. The $\Delta \mathrm{p} K_{\mathrm{a}}\left(\Delta \mathrm{p} K_{\mathrm{a}}=\mathrm{p} K_{\mathrm{a}}(\right.$ base $)-$

${ }^{a}$ Institute of Solid State Chemistry and Mechanochemistry SB RAS, Kutateladze 18, Novosibirsk, 630128, Russia.E-mail: losev.88@mail.ru,

b.zakharov@yahoo.com, eboldyreva@yahoo.com; Fax: +7 838336341 32; Tel: +783833634206

${ }^{b}$ REC-008, Novosibirsk State University, Pirogova 2, Novosibirsk, 630090, Russia $\dagger$ Electronic supplementary information (ESI) available: Includes the structural data for all the phases at multiple temperatures and pressures, the data on $\mathrm{H}$ bonds, figures presenting crystal structures, the $\mathrm{p} K_{\mathrm{a}}$ values of carboxylic acids and $\Delta \mathrm{p} K_{\mathrm{a}}$ of some glycine salts and co-crystals, and the data on cell parameters, volumes, and strain ellipsoids at multiple temperatures and pressures. CCDC 1435507, 1437082-1437089, 1437185-1437193, 1451443-1451454, and 14507161450717. For ESI and crystallographic data in CIF or other electronic format see DOI: $10.1039 /$ c6ce00561f $\mathrm{p} K_{\mathrm{a}}$ (acid)) values (measured in aqueous solution) for glycine and other listed carboxylic acids fall in the range where it is impossible to predict if the two-component crystal will be a co-crystal or a molecular salt (Table S1 and Fig. S1 in the ESI $\dagger$ ). ${ }^{25-29}$ Therefore, one might expect that the hydrogen atom of a carboxylic group of the acid in the co-crystals can be transferred from an acidic species to a basic one under pressure, similar to what has been observed for multi-component crystals of other compounds, e.g. for the 1:1 adduct formed between squaric acid and 4,4-bipyridine ${ }^{12}$ or for oxalic acid dihydrate with increasing pressure. ${ }^{30-32}$

Three glycine co-crystals with carboxylic acids have been reported to date, namely, those with glutaric, ${ }^{21,22}$ DL-tartaric $^{24}$ and phthalic ${ }^{23}$ acids (GGa, GTa, and GPh, respectively) (Fig. 1). Topologically, the crystal structures of GGa and GTa are similar, and have 3D hydrogen bond networks (Fig. 2). The crystal structure of GPh contains layers of glycine molecules, with phthalic acid molecules connected to these layers by hydrogen bonds. Weak hydrophobic stacking interactions exist between phthalic acid molecules (Fig. 2). The nature of the hydrogen bond networks is quite different in each case: GGa has only heteromolecular hydrogen bonds, GPh contains

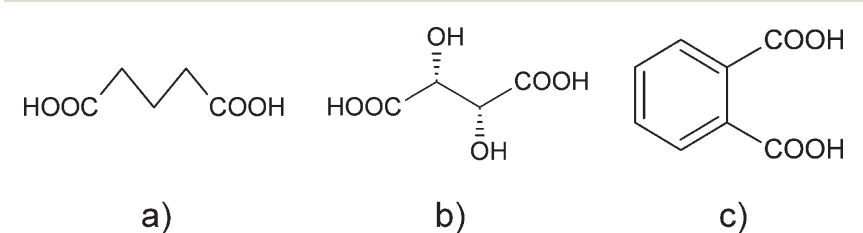

Fig. 1 Glutaric (a), DL-tartaric (b) and phthalic (c) acids. 

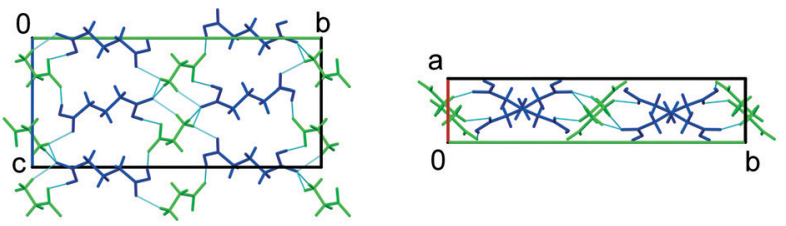

a)
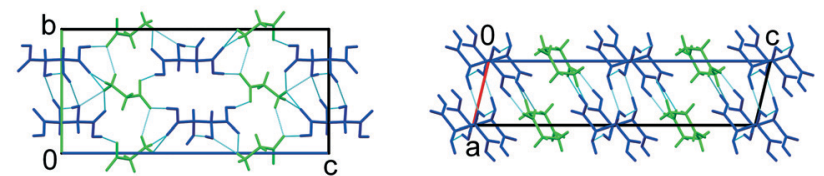

b)
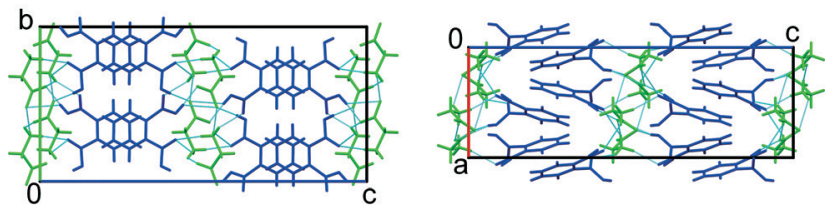

c)

Fig. 2 Comparison of the crystal structures of GGa (a), GTa (b) and GPh (c) co-crystals. Glycine - green, co-former - blue.

two types of glycine-glycine interactions (Fig. S2 in the ESI $\dagger$ ) and GTa co-crystals have both glycine-glycine and acid-acid hydrogen bonds (Fig. S2 in the ESI $\dagger$ ).

In our previous work, GGa has been shown to undergo the same structural phase transition both on cooling ${ }^{33}$ and with increasing pressure. ${ }^{34}$ This transition is related to a change in the glutaric acid conformation and occurs at about 0.1 GPa (ambient temperature), which is considerably lower than the pressure at which proton transfer could be expected, judging from the data obtained for oxalic acid dihydrate (5-6 $\mathrm{GPa}$ ) - the only multicomponent crystal constructed from neutral molecules - for which the fact of proton transfer on compression has been supported by high-quality single-crystal X-ray and neutron diffraction data, spectroscopic data, and computations. ${ }^{30,35}$ Another multicomponent glycinecontaining crystal explored at high pressure was glycinium oxalate. Spectroscopic and computational studies revealed the symmetrisation of the position of hydrogen between adjacent semi-oxalate molecules at about $8 \mathrm{GPa}^{36}$ During the phase transition in GGa, the crystal symmetry changed from monoclinic to triclinic, with every second glutaric acid molecule changing its conformation. ${ }^{33}$ The aim of the present study was to investigate co-crystals of glycine with two other co-formers, DL-tartaric and phthalic acids (GTa and GPh, respectively), and to compare their response to variations in temperature and pressure with that of GGa. We also compared the response of GGa, GTa and GPh to pressure with that of selected amino acids salts.

\section{Experimental section}

\section{Materials}

All reagents were commercially available and were used without further purification. The following chemicals were used: $\alpha$-glycine (Reactiv, Russia, pur. >95\%), DL-tartaric acid (Reactiv, Russia, pur. >95\%), phthalic acid (Sigma-Aldrich, puriss $\geq 99.5 \%$ ), and distilled water.

\section{Crystallisation}

GTa and GPh single crystals were grown from equimolar aqueous solutions of the starting components (total solid mass $\sim 0.6 \mathrm{~g}$ ). Particular attention was required for the successful preparation of GPh crystals, and a three-step process was developed: 1. preparation of a saturated solution of $\alpha$-glycine at room temperature; 2 . addition of phthalic acid up to a saturation limit at approximately $70-80{ }^{\circ} \mathrm{C} ; 3$. slow cooling to room conditions, upon which GPh crystallised.

\section{Single crystal X-ray diffraction}

Low-temperature experiments. Variable-temperature single-crystal X-ray diffraction was performed using a STOE IPDS-2 diffractometer with an image plate detector, graphite monochromator, Mo K $\alpha$ radiation and an Oxford Cryostream cooling device. The temperature range was $300-100 \mathrm{~K}$ with a step size of $25 \mathrm{~K}$ and a cooling rate of $100 \mathrm{~K} \mathrm{~h}^{-1}$. The sample was fixed to the holder by using LV CryoOil (MiTeGen).

$\mathrm{X}^{-\mathrm{AREA}^{37}}$ and X-RED32 (ref. 37) were used for cell refinement, data collection and data reduction. The crystal structures were solved by direct methods using SHELXS97 (ref. 38) and refined on $F^{2}$ using X-STEP, ${ }^{37}$ SHELXL2014 (ref. 38) and OLEX2 Version 1.2.7. ${ }^{39}$ Mercury ${ }^{40}$ was used for the visualisation and analysis of structure motifs. Win_Strain ${ }^{41}$ was used to calculate the linear strain along the principal axes of the strain ellipsoid. All structures were deposited with the Cambridge Crystallographic Data Centre (CCDC numbers 1435507, 1437082-1437089 and 1437185-1437193 for GTa and GPh, respectively) and are available at http://www.ccdc. cam.ac.uk/.

High-pressure experiments. Hydrostatic pressure was generated in an Almax-Boehler diamond anvil cell (DAC). ${ }^{42}$ Natural diamonds were used without a beryllium backing plate; this was suitable for both X-ray diffraction and Raman experiments. A steel gasket with an initial thickness of $200 \mu \mathrm{m}$ was preindented to $\sim 100 \mu \mathrm{m}$. The hole was $280 \mu \mathrm{m}$ in diameter. The ruby fluorescence method was used for pressure calibration, ${ }^{43,44}$ with a precision of $0.05 \mathrm{GPa}$. An equivoluminar mixture of pentane and iso-pentane was used as the pressuretransmitting medium.

The data were collected (pressure range for GTa: 0.1-5.9 GPa, for GPh: 0.2-6.0 GPa) using an Oxford Diffraction Gemini R Ultra X-ray diffractometer with a CCD area detector and Mo K $\alpha$ radiation. The following software packages were used: CrysAlisPro $^{45}$ (data collection, cell refinement and data reduction), SHELXS 97 (structure solution), ${ }^{38}$ SHELXL 2014, ${ }^{38}$ X-STEP 32 (ref. 37) (structure refinement) and OLEX2 Version 1.2.7 (structure refinement). ${ }^{39}$ The absorption of X-rays by the diamonds was accounted for using ABSORB (Gaussian absorption correction). ${ }^{46}$ Reflections from the sample that overlapped with those from the diamond and gasket were 
excluded manually. Mercury ${ }^{40}$ was used for the structure visualisation and analysis.

The crystal structure of the GTa high-pressure phase was solved by direct methods implemented in SIR2014 (ref. 47) software. All atoms in the structure of GTa in the DAC experiments were refined isotropically since not enough reflections were obtained from the dataset for adequate anisotropic refinement. The hydrogen atom parameters for all DAC experiments were constrained and refined using a riding model (AFIX 33 with $U_{\text {iso }}(\mathrm{H})=1.2 U_{\text {eq }}(\mathrm{N})$ and $\mathrm{NH}$ distances equal to $0.890 \AA$ for $\mathrm{NH}_{3}$ groups; AFIX 83 with $U_{\text {iso }}(\mathrm{H})=1.2 U_{\text {eq }}(\mathrm{O})$ and $\mathrm{OH}$ distances equal to $0.820 \AA$ for $\mathrm{O}-\mathrm{H}$ groups, AFIX 13 with $U_{\text {iso }}(\mathrm{H})=1.2 U_{\text {eq }}(\mathrm{C})$ and $\mathrm{CH}$ distances equal to $0.980 \AA$ for $\mathrm{CH}$ groups, AFIX 23 with $U_{\text {iso }}(\mathrm{H})=1.2 U_{\text {eq }}(\mathrm{C})$ and $\mathrm{CH}$ distances equal to $0.970 \AA$ for $\mathrm{CH}_{2}$ groups, AFIX 43 with $U_{\text {iso }}(\mathrm{H})=$ $1.2 U_{\text {eq }}(\mathrm{C})$ and $\mathrm{CH}$ distances equal to $0.930 \AA$ for aromatic $\mathrm{CH}$ groups). All structures were deposited with the Cambridge Crystallographic Data Centre (CCDC numbers 14514431451454 and 1450716-1450717 for GTa and GPh, respectively) and are available at http:/www.ccdc.cam.ac.uk/.

\section{Raman spectroscopy}

Raman experiments were performed in parallel with X-ray diffraction to obtain additional information on high-pressure structural transformations. For each pressure point, Raman spectra were recorded immediately after collection of X-ray diffraction data. Single-crystal Raman spectra were collected using a LabRAM Horiba single spectrometer with a CCD Symphony (Jobin Yvon) detector. The $488 \mathrm{~nm}$ line of an $\mathrm{Ar}^{+}$laser with a beam size of $1 \mathrm{~mm}$ and $8 \mathrm{~mW}$ power was used for spectral excitation. The spectra at all pressures were measured in $180^{\circ}$ backscattering collection geometry using a Raman microscope. While the spectral resolution was $2 \mathrm{~cm}^{-1}$, the observed broadening was significantly higher due to an increase in anharmonicity with increasing pressure.

\section{Optical microscopy}

Microphotography was performed using a Nikon AZ100 optical microscope and the above-mentioned Raman microscope.

\section{Results and discussion}

The parameters characterising the data collection and refinement, as well as the crystal data at different temperatures and pressures are summarised in Tables ST1-ST8 in the ESI. $\dagger$ In contrast with GGa, GTa and GPh were stable on cooling from $300 \mathrm{~K}$ to $100 \mathrm{~K}$, during which monotonic changes to the volume and cell parameters were observed. There was no evidence of phase transitions over this temperature range (Fig. $\mathrm{S} 3$ and S4 in the ESI $\dagger$ ). The volume decrease from $293 \mathrm{~K}$ to $100 \mathrm{~K}$ was slightly higher for GPh $(2.6 \%)$ compared with that for GTa (2.2\%). For comparison, a low-temperature reversible first-order phase transition, which was previously observed in GGa between 225 and $200 \mathrm{~K}$, was associated with a volume change of approximately $1 \%$ compared to the volume at 300 K. Most probably, the conformational flexibility of glutaric acid is a reason for the phase transition in GGa at low temperature. In the case of GTa, a skeleton of DL-tartaric acid is stabilised due to hydrogen bonds between hydroxyl groups, whereas in the structure of GPh there are also multiple interactions between the aromatic rings of the phthalic acids and glycine layers that allow more compression compared with GGa.

Judging from the almost equal values of the two $\mathrm{C}-\mathrm{O}$ distances in the carboxylic group of glycine, the group remained non-protonated, i.e. no proton migration from the co-former - a carboxylic acid - to glycine occurred on cooling over the whole studied temperature range. The main structural changes were related to compression or extension of hydrogen bonds. The data on the geometries of the hydrogen bonds are summarised in Tables ST5-ST8 in the ESI. $\dagger$ Most of the hydrogen bonds in GTa and GPh shorten on cooling, whereas only one of them (O3-H3 $\cdots \mathrm{O} 2[x-1, y, z]$ in GTa) extends slightly (by $0.4 \%$, Fig. S5 and S6 in the ESI $\dagger$ ). Interestingly, the $\mathrm{O} 3-\mathrm{H} 3 \cdots \mathrm{O} 2[x-1, y, z]$ hydrogen bond is one of the shortest (and, supposedly, the strongest) intermolecular interactions in GTa (Fig. S5 and S6 in the ESI†). Evaluation of all torsion angles in the structure of GTa reveals a maximal change of about $2^{\circ}$, which could be sufficient to make the hydrogen bond longer. Alternatively, the bond extension can be related to a slight change of the molecular coordinates (shifting the molecule as a whole) with temperature, or to the cooperative effect of the compression of other hydrogen bonds in the 3D network, which accounts for the lowering of the overall lattice energy. Analysis of the linear strain in the two glycine co-crystals on cooling shows two different types of behavior of GTa and GPh crystal structures: GTa has one preferential direction of compression, whereas the compression of GPh along two principal axes is approximately equivalent. The maximum compression in the GTa structure is close to the direction of the mixed glycine-DL-tartaric acid hydrogen bonded chains. The maximum linear compression in the GPh structure lies along the stacks of the phthalic acid molecules, where weak hydrophobic interactions dominate. Another direction of the significant compression in GPh lies along the $c$-axis perpendicular to glycine layers and almost parallel with respect to the plane to which the benzene rings of phthalic acid are slightly inclined. The presence of nonhydrogen bonded layers of phthalic acid results in the appearance of two almost equivalent directions of compression (Fig. S7-S9 in the ESI $\dagger$ ).

With increasing pressure, both GTa and GPh underwent reversible first-order phase transitions accompanied by fracture (Fig. 3). These transitions occurred at very low pressures (about 0.5 GPa and $1 \mathrm{GPa}$, respectively). For GTa, changes in the cell parameters and volume were tracked (Fig. 4), and the structure of the high-pressure phase was solved. The change from a monoclinic space group $\left(P 2_{1} / c\right)$ to a triclinic one $(P \overline{1})$ was accompanied by the doubling of the number of formula units per asymmetric unit cell $\left(Z^{\prime}\right)$ (Fig. 5), similar to what has been observed in the case of GGa (Fig. S10 in the ESI $\dagger$ ). The increase in $Z^{\prime}$ in the denser phases formed on cooling or 


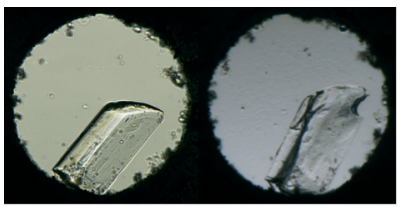

$0.1 \mathrm{GPa} \longrightarrow 0.4 \mathrm{GPa}$

a)

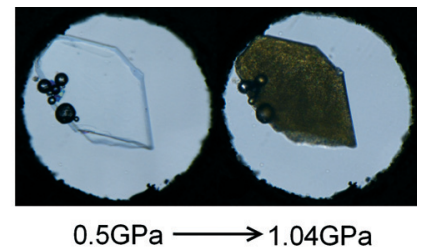

b)
Fig. 3 Images of co-crystals in the DAC. (a) Fracture of the glycine-DLtartaric acid (GTa) co-crystals and (b) glycine-phthalic acid (GPh) cocrystals across the high-pressure phase transition.
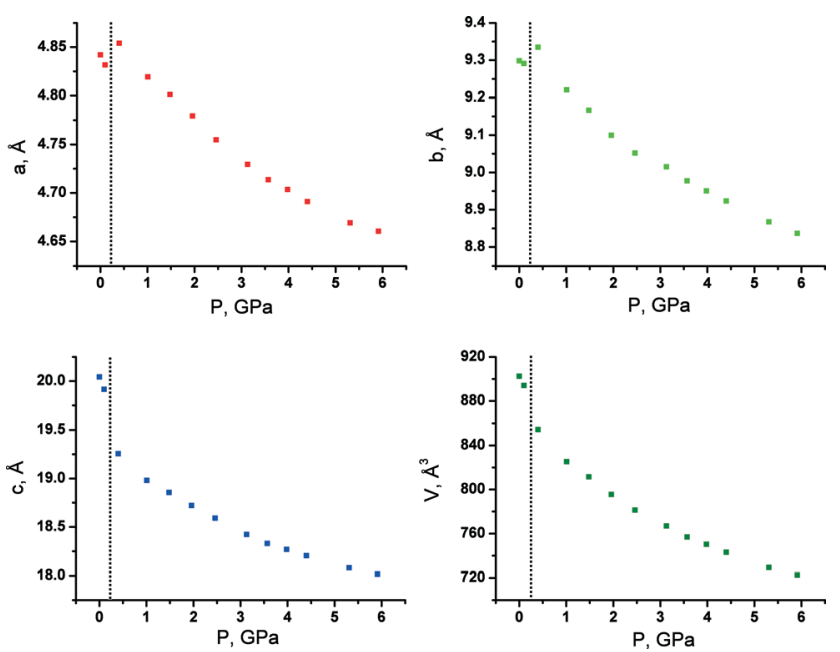

Fig. 4 Unit cell parameters and volume of GTa at different pressures. The phase transition takes place between 0.1 and $0.4 \mathrm{GPa}$.
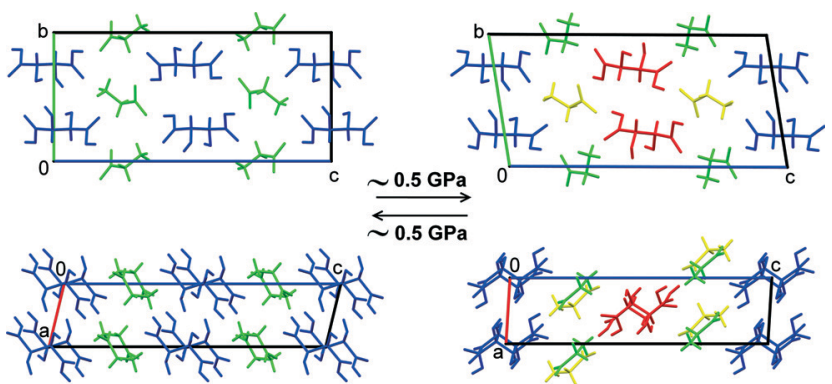

$\mathrm{P} 2{ }_{1} / \mathrm{c}$

$\mathrm{P} 1$

Fig. 5 Structural changes in GTa due to the phase transition at high pressure. Different colours mark symmetry-independent molecules.

at high pressures has been reported earlier also for singlecomponent molecular crystals, ${ }^{48}$ e.g. for chlorpropamide, ${ }^{49,50}$ although examples of the opposite are also known. ${ }^{51}$ Anisotropy of the linear strain and the directions of the principal axes of the strain ellipsoid for the GTa triclinic phase have been calculated in the range from 0.4 to $5.91 \mathrm{GPa}$ (Table S2 and Fig. S11a in the ESI $\dagger$ ). The triclinic phase of GTa contains two symmetrically independent dimeric chains of DL-tartaric acid that are stabilised by $\mathrm{O}-\mathrm{H} \cdots \mathrm{O}$ hydrogen bonds between side $\mathrm{OH}$-groups. An increase in the pressure causes the sliding of the tartaric acids relative to each other. The distance between $\mathrm{O} 13$ and $\mathrm{O} 16[2-x, 2-y, 2-z]$ becomes shorter and at about $3 \mathrm{GPa}$ reaches a value, which is usually assumed to be the threshold distance, below which an interaction can be classified as a hydrogen bond (about $2.8 \AA$ at $5.9 \mathrm{GPa}) .{ }^{52}$ Though the coordinates of the H-atoms cannot be considered as reliable, based on the $\mathrm{O}-\mathrm{O}$ distances one can suppose that the $\mathrm{O}-\mathrm{H} \cdots \mathrm{O}$ hydrogen bond becomes three-centered (Fig. S12 in the ESI $\dagger$ ). Similar changes in the hydrogen bonds in the crystals of amino acids and their salts have been reported earlier for DL-alaninium semi-oxalate monohydrate, ${ }^{3}$ L-serine, ${ }^{53}$ and DL-serine. ${ }^{54}$ Another major structural change is associated with the coordination of one of the glycine molecules: as pressure increases, the N11-O11 $[2-x, 1-y, 2-z]$ distance shortens, which can be related to the strengthening of the interaction between glycine and the corresponding tartaric acid molecules (Fig. S13 in the ESI $\dagger$ ).

This pressure-induced phase transition is similar to the ones observed earlier in GGa, both on cooling ${ }^{33}$ and with increasing pressure (Fig. S10 in the ESI $\dagger$ ). ${ }^{34}$ As for the intriguing question regarding high-pressure proton transfer and an associated co-crystal-salt transition, experimental limitations did not offer sufficient precision to determine the proton positions within experimental error. That said, the pressure at which such a transfer might be expected (based on data for oxalic acid dihydrate) has not been reached, and future work can address this question more deeply. An attempt to record the Raman spectra of the single crystal in the DAC was not successful, with the laser beam decomposing the crystal despite the use of attenuating filters (Fig. S14 in the ESI $\dagger$ ). Thus, information regarding proton transfer at high pressure was not obtainable.

The pressure-induced phase transition in GPh had an induction period of a few hours, so that the low-pressure phase could be maintained for a period of time at high pressure (over-pressurised ambient-pressure phase of GPh), after which the crystal was suddenly completely destroyed as a result of the phase transition into the high-pressure phase (Fig. 3b). The fragmentation of GPh rendered the collection of X-ray diffraction data suitable for structure solution impossible. It also should be mentioned that the initial crystal of GPh didn't undergo destruction and phase transition at 0.19 and $0.50 \mathrm{GPa}$. At each of these two points, the total time during which the crystal was kept at a constant pressure was about 42 hours (spectroscopic and X-ray diffraction experiments) and no phase transitions were detected. Tentative conclusions on the structural changes across the phase transition were made based on the analysis of the original ambient-pressure crystal structure (Fig. 2c) and on the Raman spectra (which, in contrast to GPh, could be collected as the crystals did not burn) (Fig. 6). The rationale for the destructive nature of the phase transition rests on a dramatic change in the molecular packing. The change in the unit cell parameters before phase transition shows that the maximum compression occurs along the $a$ - and $c$-axes (Fig. S11b in the ESI $\dagger$ ). In this case, one might suggest that a steric conflict 

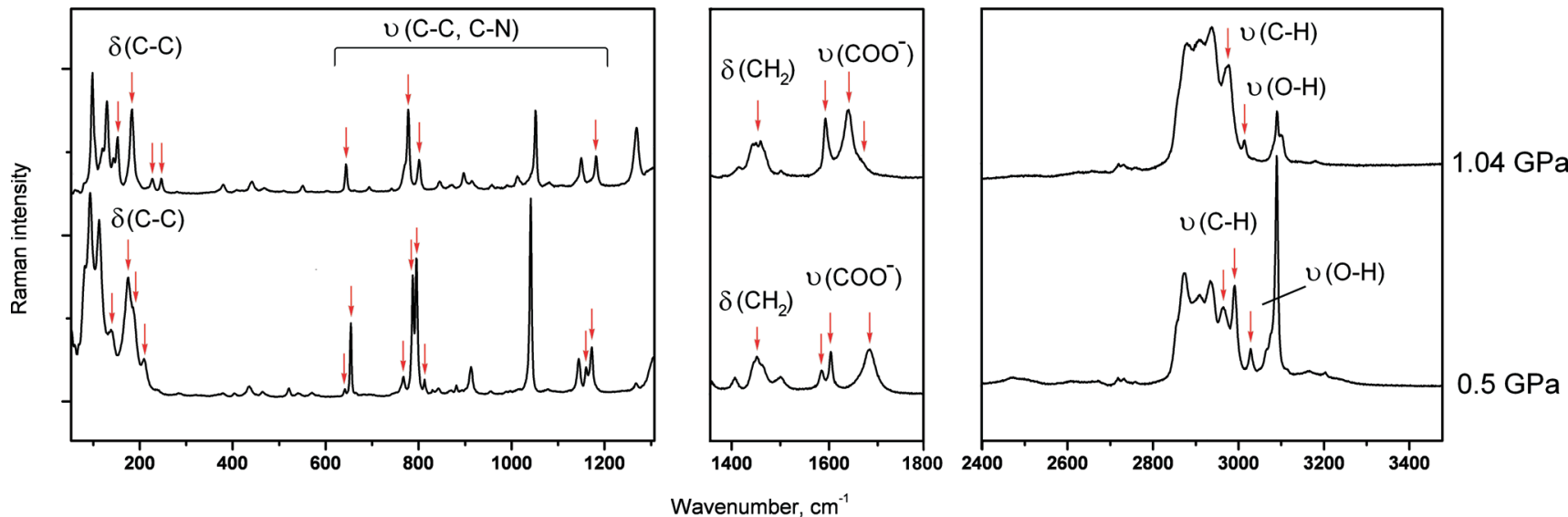

Fig. 6 Raman spectra of the two forms of a glycine-phthalic acid co-crystal (GPh) at different pressures; before and after the phase transition. Major changes are shown by red arrows. $v$-Stretching vibrations, $\delta$-deformation vibrations.

arises on compression due to shortening of the distances either between the aromatic rings of the phthalic acid and the glycine layers (compression along $c$ ), or between the aromatic rings (compression along $a$ ). One can suggest that these steric conflicts may result in shifts of the aromatic rings with respect to each other, such that a double layer is substituted for a single one. The Raman spectra changed across the phase transition, both in the low frequency range and in the range of vibrations of hydrogen bonded groups. The shift in band frequencies corresponding to the $\mathrm{O}-\mathrm{H}$ and $\mathrm{N}-\mathrm{H}$ stretching vibrations is not significant, supporting the idea that changes in the strengths of the hydrogen bonds across the phase transition are only minor (Fig. 6). This supports the hypothesis that major structural changes are related to stacking interactions of the aromatic rings, and not of the hydrogen bond network. The long induction period, the overpressurising of the low-pressure phase prior to the phase transition and the spontaneous rapid propagation of the latter suggest a cooperative mechanism of the transformation, with slow nucleation but rapid nuclei growth, similar to the ones reported earlier for pressure-induced $\gamma \rightarrow \delta$ glycine, ${ }^{55}$ L-serine-I $\rightarrow$ L-serine-II, $^{56}$ or $\beta$-alanine-II $\rightarrow \beta$-alanine-V ${ }^{57}$ phase transitions at high pressure.

\section{Conclusions}

To date, all three known glycine-carboxylic acid co-crystals have been investigated across a wide range of temperatures (293-100 K) and pressures (0-6 GPa). All three structures undergo phase transitions at relatively low pressures (below 1 GPa), but only the glycine-glutaric acid co-crystal shows a phase transition on cooling. ${ }^{33}$ The data obtained for the glycine-glutaric acid and glycine-DL-tartaric acid co-crystals suggest that if a crystal structure includes both conformationally flexible (carboxylic acid) and rigid (glycine) components, linked in a 3D-network of hydrogen bonds, then the pressure-induced phase transition is related to conformational changes of the flexible species. If the flexible fragment is immobilised by intra- and intermolecular hydrogen bonds in the crystal structure (as in the dimer of tartaric acid), then its conformation can be preserved in the course of the phase transition. Additionally, slight changes in the position of the entire species may also be involved in such transformations. Overall, the corresponding rearrangement of the crystal structure is relatively small, and the crystals can withstand the applied mechanical stresses. In cases where the structure is formed by two rigid species (e.g. in glycine-phthalic acid cocrystals), with a robust 3D network of hydrogen bonds, changes in the crystal structure may be significantly larger and the crystal is destroyed. Similar effects have been reported for bis-glycinium oxalate. ${ }^{58-60}$

\section{Acknowledgements}

This work was supported by the Russian Foundation for Basic Research (RFBR) (Grants No. 14-03-31866 mol_a and 16-3360089 mol_a_dk), and RAS (Project 44.3.4).

\section{References}

1 E. V. Boldyreva, Z. Kristallogr., 2014, 229, 236.

2 B. A. Zakharov, B. A. Kolesov and E. V. Boldyreva, Phys. Chem. Chem. Phys., 2011, 13, 13106.

3 B. A. Zakharov and E. V. Boldyreva, Acta Crystallogr., Sect. B: Struct. Sci., Cryst. Eng. Mater., 2013, 69, 271.

4 B. A. Zakharov and E. V. Boldyreva, J. Mol. Struct., 2014, 1078, 151.

5 A. K. Mishra, C. Murli, N. Garg, R. Chitra and S. M. Sharma, J. Phys. Chem. B, 2010, 114, 17084.

6 V. S. Minkov, E. V. Boldyreva, T. N. Drebushchak and C. H. Görbitz, CrystEngComm, 2012, 14, 5943.

7 I. Majerz, Z. Malarski and L. Sobczyk, Chem. Phys. Lett., 1997, 274, 361.

8 T. Steiner, I. Majerz and C. C. Wilson, Angew. Chem., Int. Ed., 2001, 40, 2651.

9 C. C. Wilson, Acta Crystallogr., Sect. B: Struct. Sci., 2001, 57, 435. 
10 J. A. Cowan, J. A. Howard, G. J. McIntyre, S. M. Lo and I. D. Williams, Acta Crystallogr., Sect. B: Struct. Sci., 2003, 59, 794.

11 P. Macchi, N. Casati, W. G. Marshall and A. Sironi, CrystEngComm, 2010, 12, 2596.

12 D. M. S. Martins, D. S. Middlemiss, C. R. Pulham, C. C. Wilson, M. T. Weller, P. F. Henry, N. Shankland, K. Shankland, W. G. Marshall, R. M. Ibberson, K. Knight, S. Moggach, M. Brunelli and C. A. Morrison, J. Am. Chem. Soc., 2009, 131, 3884.

13 F. H. Allen, Acta Crystallogr., Sect. B: Struct. Sci., 2002, 58, 380.

14 N. A. Tumanov, E. V. Boldyreva and N. E. Shikina, Acta Crystallogr., Sect. C: Cryst. Struct. Commun., 2010, 66, 0279.

15 M. Subha Nandhini, R. V. Krishnakumar and S. Natarajan, Acta Crystallogr., Sect. C: Cryst. Struct. Commun., 2001, 57, 115.

16 R. Chitra, V. Thiruvenkatam, R. R. Choudhury, M. V. Hosur and T. N. Guru Row, Acta Crystallogr., Sect. C: Cryst. Struct. Commun., 2006, 62, 0274.

17 E. A. Losev, B. A. Zakharov, T. N. Drebushchak and E. V. Boldyreva, Acta Crystallogr., Sect. C: Cryst. Struct. Commun., 2011, 67, 0297.

18 K. Rajagopal, R. V. Krishnakumar, A. Mostad and S. Natarajan, Acta Crystallogr., Sect. E: Struct. Rep. Online, 2001, 57, o751.

19 L. Zong-Sheng and C. Jian-She, Acta Crystallogr., Sect. E: Struct. Rep. Online, 2007, 63, 02857.

20 A. Thirunavukkarasu, A. Silambarasan, R. M. Kumar, P. R. Umarani and G. Chakkaravarthi, Acta Crystallogr., Sect. E: Struct. Rep. Online, 2014, 70, 0397.

21 E. A. Losev, B. A. Zakharov, T. N. Drebushchak and E. V. Boldyreva, Acta Crystallogr., Sect. C: Cryst. Struct. Commun., 2011, 67, 0297.

22 B. Riscob, M. Shakir, J. K. Sundar, S. Natarajan, M. A. Wahab and G. Bhagavannarayana, Spectrochim. Acta, Part A, 2011, 78, 543.

23 T. Balakrishnan, K. Ramamurthi and S. Thamotharan, Acta Crystallogr., Sect. E: Struct. Rep. Online, 2013, 69, 057.

24 T. Mohandas, C. R. D. Inbaseelan, S. Saravanan and P. Sakthivel, Acta Crystallogr., Sect. E: Struct. Rep. Online, 2013, 69, o236.

25 S. L. Childs, G. P. Stahly and A. Park, Mol. Pharmaceutics, $2007,4,323$.

26 V. Stilinović and B. Kaitner, Cryst. Growth Des., 2012, 12, 5763.

27 G. Ramon, K. Davies and L. R. Nassimbeni, CrystEngComm, 2014, 16, 5802.

28 A. Lemmerer, S. Govindraju, M. Johnston, X. Motloung and K. L. Savig, CrystEngComm, 2015, 17, 3591.

29 A. J. Cruz-Cabeza, CrystEngComm, 2012, 14, 6362.

30 N. Casati, P. Macchi and A. Sironi, Chem. Commun., 2009, 2679.

31 N. Casati, NATO Science for Peace and Security Series B: Physics and Biophysics, 2010, p. 161.

32 P. Macchi, N. Casati, W. G. Marshall and A. Sironi, CrystEngComm, 2010, 12, 2596.
33 B. A. Zakharov, E. A. Losev, B. A. Kolesov, V. A. Drebushchak and E. V. Boldyreva, Acta Crystallogr., Sect. B: Struct. Sci., 2012, 68, 287.

34 B. A. Zakharov, E. A. Losev and E. V. Boldyreva, CrystEngComm, 2013, 15, 1693.

35 H. Bhatt, A. K. Mishra, C. Murli, A. K. Verma, N. Garg, M. N. Deo and S. M. Sharma, Phys. Chem. Chem. Phys., 2016, 18, 8065.

36 H. Bhatt, C. Murli, A. K. Mishra, A. K. Verma, N. Garg, M. N. Deo, R. Chitra and S. M. Sharma, J. Phys. Chem. B, 2016, 120, 851.

37 Stoe and Cie, $X$-AREA and $X$-RED32, Stoe and Cie, Darmstadt, Germany, 2006.

38 G. M. Sheldrick, Acta Crystallogr., Sect. C: Struct. Chem., 2015, 71, 3 .

39 O. V. Dolomanov, L. J. Bourhis, R. J. Gildea, J. A. K. Howard and H. Puschmann, J. Appl. Crystallogr., 2009, 42, 339.

40 C. F. Macrae, I. J. Bruno, J. A. Chisholm, P. R. Edgington, P. McCabe, E. Pidcock, L. Rodriguez-Monge, R. Taylor, J. van de Streek and P. A. Wood, J. Appl. Crystallogr., 2008, 41, 466.

41 R. J. Angel, Win_Strain, 2011, http://www.rossangel.com.

42 R. Boehler, Rev. Sci. Instrum., 2006, 77, 115103.

43 R. A. Forman, G. J. Piermarini, J. D. Barnett and S. Block, Science, 1972, 176, 284.

44 G. J. Piermarini, S. Block, J. D. Barnett and R. A. Forman, J. Appl. Phys., 1975, 46, 2774.

45 Oxford Diffraction, CrysAlis Pro, Oxford Diffraction Ltd, Abington, England, 2010.

46 R. J. Angel, J. Appl. Crystallogr., 2004, 37, 486.

47 M. C. Burla, R. Caliandro, B. Carrozzini, G. L. Cascarano, C. Cuocci, C. Giacovazzo, M. Mallamo, A. Mazzone and G. Polidori, J. Appl. Crystallogr., 2015, 48, 306.

48 K. M. Steed and J. W. Steed, Chem. Rev., 2015, 115, 2895.

49 T. N. Drebushchak, V. A. Drebushchak and E. V. Boldyreva, Acta Crystallogr., Sect. B: Struct. Sci., 2011, 67, 163.

50 Y. V. Seryotkin, T. N. Drebushchak and E. V. Boldyreva, Acta Crystallogr., Sect. B: Struct. Sci., Cryst. Eng. Mater., 2013, 69, 77.

51 R. D. L. Johnstone, M. Leva, A. R. Lennie, H. McNab, E. Pidcock, J. E. Warren and S. Parsons, CrystEngComm, 2010, 12, 2520.

52 G. A. Jeffrey, An Introduction to Hydrogen Bonding, Oxford University Press, Oxford, 1997, p. 12.

53 T. N. Drebushchak, H. Sowa, Y. V. Seryotkin, E. V. Boldyreva and H. Ahsbahs, Acta Crystallogr., Sect. E: Struct. Rep. Online, 2006, 62, 04052.

54 B. A. Zakharov, B. A. Kolesov and E. V. Boldyreva, Acta Crystallogr., Sect. B: Struct. Sci., 2012, 68, 275.

55 E. V. Boldyreva, Phase Transitions, 2009, 82, 303.

56 E. V. Boldyreva, H. Sowa, Yu. V. Seryotkin, T. N. Drebushchak, H. Ahsbahs, V. V. Chernyshev and V. P. Dmitriev, Chem. Phys. Lett., 2006, 429, 474.

57 B. A. Zakharov, N. A. Tumanov and E. V. Boldyreva, CrystEngComm, 2015, 17, 2074.

58 H. Bhatt, C. Murli, N. Garg, M. N. Deo, R. Chitra, R. R. Choudhury and S. M. Sharma, Chem. Phys. Lett., 2012, 532, 57. 
59 A. K. Mishra, C. Murli, N. Garg, R. Chitra and S. M. Sharma, J. Phys. Chem. B, 2010, 114, 17084.
60 H. Bhatt, C. Murli, N. Garg, A. K. Mishra, M. N. Deo and S. M. Sharma, AIP Conf. Proc., 2014, 1591, 230. 